\title{
Oxygen-activated growth and bandgap tunability of large single-crystal bilayer graphene
}

\section{Citation}

Hao, Yufeng, Lei Wang, Yuanyue Liu, Hua Chen, Xiaohan Wang, Cheng Tan, Shu Nie, et al. 2016. "Oxygen-Activated Growth and Bandgap Tunability of Large Single-Crystal Bilayer Graphene." Nature Nanotechnology 11 (5) (February 1): 426-431. doi:10.1038/nnano.2015.322.

\section{Published Version}

doi:10.1038/nnano.2015.322

\section{Permanent link}

http://nrs.harvard.edu/urn-3:HUL.InstRepos:34310079

\section{Terms of Use}

This article was downloaded from Harvard University's DASH repository, and is made available under the terms and conditions applicable to Other Posted Material, as set forth at http:// nrs.harvard.edu/urn-3:HUL.InstRepos:dash.current.terms-of-use\#LAA

\section{Share Your Story}

The Harvard community has made this article openly available.

Please share how this access benefits you. Submit a story.

\section{Accessibility}




\title{
Oxygen-Activated Growth and Bandgap Tunability of Large Single-Crystal Bilayer Graphene
}

\author{
Yufeng Hao, ${ }^{1}$ Lei Wang, ${ }^{1}$ Yuanyue Liu, ${ }^{2,3}$ Hua Chen, ${ }^{4}$ Xiaohan Wang, ${ }^{5}$ Cheng Tan, ${ }^{1}$ Shu \\ Nie, ${ }^{6}$ Ji Won Suk, ${ }^{7}$ Tengfei Jiang, ${ }^{8}$ Tengfei Liang, ${ }^{9}$ Junfeng Xiao, ${ }^{1}$ WenjingYe, ${ }^{9}$ Cory R. \\ Dean, ${ }^{10}$ Boris I. Yakobson, ${ }^{2}$ Kevin F. McCarty, ${ }^{6}$ Philip Kim, ${ }^{11}$ James Hone, ${ }^{1 *}$ Luigi \\ Colombo, ${ }^{12^{*}}$ Rodney S. Ruoff ${ }^{13,5^{*}}$
}

${ }^{1}$ Department of Mechanical Engineering, Columbia University, New York, New York, 10027, USA

${ }^{2}$ Department of Materials Science and NanoEngineering, Rice University, Houston, Texas, 77005, USA

${ }^{3}$ National Renewable Energy Laboratory, Golden, Colorado, 80401, USA

${ }^{4}$ Department of Physics, The University of Texas at Austin, Austin, Texas, 78712, USA

${ }^{5}$ Department of Mechanical Engineering and the Materials Science and Engineering Program,

The University of Texas at Austin, Austin, Texas, 78712, USA

${ }^{6}$ Sandia National Laboratories, Livermore, California, 94550, USA

${ }^{7}$ School of Mechanical Engineering, Sungkyunkwan University, Suwon, 440-746, Korea

${ }^{8}$ Department of Materials Science and Engineering, University of Central Florida, Orlando, Florida, 32816, USA

${ }^{9}$ Department of Mechanical Engineering, Hong Kong University of Science and Technology, Clear Water Bay, Kowloon, Hong Kong, China

${ }^{10}$ Department of Physics, Columbia University, New York, New York, 10027, USA

${ }^{11}$ Department of Physics, Harvard University, Cambridge, Massachusetts, 02138, USA

${ }^{12}$ Texas Instruments, Dallas, Texas, 75243, USA

${ }^{13}$ Center for Multidimensional Carbon Materials, Institute for Basic Science, Ulsan National Institute of Science and Technology, Ulsan, 689-798, Korea

*Corresponding Authors: R.S.R. (ruofflab@gmail.com), L.C. (colombo@ti.com), and J.H. (jh2228@columbia.edu). 
Distinct from zero-bandgap single-layer graphene (SLG), Bernal (AB)-stacked bilayer graphene (BLG) is a semiconductor whose bandgap can be tuned by a transverse electric field, making it a unique material for a number of electronic and photonic devices ${ }^{1-3}$. For this purpose, a scalable approach for the synthesis of high-quality BLG is critical, which requires minimizing the number of crystalline defects in both graphene layers, such as domain boundaries ${ }^{4,5}$, and maximizing the area of Bernal stacking, which is necessary for bandgap tunability ${ }^{6}$. Many attempts have thus been made to search for the optimal growth conditions of single-crystal $\mathrm{BLG}$ on $\mathrm{Cu}$, $\mathrm{Ni}$, or $\mathrm{Cu}-\mathrm{Ni}$ alloys ${ }^{7-19}$, but these have only resulted in small BLG domains with large variations in domain size from lab to lab under presumably similar growth conditions, and no control over the stacking order. Here we demonstrate that in an oxygen (O)-activated chemical vapor deposition (CVD) process, half-millimeter size, Bernal-stacked, BLG single crystals can be synthesized on Cu surfaces. Besides the traditional "surface-limited" growth mechanism for SLG ( $1^{\text {st }}$ layer), we discovered new microscopic steps governing the growth of the $2^{\text {nd }}$ graphene layer below the $1^{\text {st }}$ layer as the diffusion of carbon $(\mathrm{C})$ atoms through the $\mathrm{Cu}$ bulk after complete dehydrogenation of hydrocarbon molecules on the $\mathrm{Cu}$ surface, which does not occur in the absence of $\mathrm{O}$. Moreover, we found that the efficient diffusion of the $\mathrm{C}$ atoms present at the interface between $\mathrm{Cu}$ and the $\mathbf{1}^{\text {st }}$ graphene layer further facilitates growth of large domains of the $2^{\text {nd }}$ layer. The CVD BLG reveals superior electrical quality, with the device on/off ratio greater than $10^{4}$, and a tunable bandgap up to $\sim 100 \mathrm{meV}$ at a displacement field of 0.9 V/nm.

A $\mathrm{Cu}$ pocket, formed by folding either an O-rich $\mathrm{Cu}(\mathrm{OR}-\mathrm{Cu})$ or an $\mathrm{O}$-free $\mathrm{Cu}(\mathrm{OF}-\mathrm{Cu})$ foil $^{20}$ and crimping the open edges, is used to study BLG growth (Fig. 1a and Supplementary Fig. 
1). Gas exchange between the interior and exterior of the pocket is limited to 200-400 nm gaps at the crimped edges (Fig. 1a, inset), which is more than 3 orders of magnitude smaller than the mean free path of the $\mathrm{CH}_{4}$ under typical growth conditions. The $\mathrm{CH}_{4}$ therefore reaches the interior through Knudsen diffusion (details in Supplementary Information) with a diffusivity of about 4 orders of magnitude lower than that in the ambient outside the pocket. Monte Carlo simulations and analytical calculations show that when $\mathrm{CH}_{4}$ is introduced into the chamber, it takes a few minutes to reach equilibrium between the inside and outside of the pocket.(Supplementary Fig. 3)

After 6 hours of growth on OR-Cu, the exterior surface is completely covered with graphene, whereas on the interior surface only isolated dendritic graphene islands are formed with a density of $\sim 0.5 \mathrm{~mm}^{-2}$ (Fig. 1b), much lower than that on the outside, leaving more than $60 \%$ of the $\mathrm{Cu}$ surface exposed. Most interestingly, on the exterior surface there are large (300-550 $\mu \mathrm{m})$ BLG domains with a high density $\left(\sim 3 \mathrm{~mm}^{-2}\right)$ (Fig. 1c). The compact hexagonal shape of the BLG domains indicates different growth kinetics from the dendritic SLG islands on the interior surface. We further found that a low $\mathrm{P}_{\mathrm{CH} 4}\left(\sim 10^{-3}\right.$ Torr $)$ is important for $2^{\text {nd }}$ layer nucleation and growth, while thicker layers are efficiently suppressed.

Low energy electron diffraction (LEED) characterization showed that the $2^{\text {nd }}$ layer graphene domains are single-crystal and grow beneath the continuous $1^{\text {st }}$ layer (Supplementary Fig. 14). Large-scale Raman mapping of the $2 \mathrm{D}$ band after transfer to $\mathrm{Si} / \mathrm{SiO}_{2}$ showed that more than $80 \%$ of the BLG regions are Bernal stacked ${ }^{21}$ (Fig. 1d). The stacking order was further confirmed by transmission electron microscopy (Supplementary Fig. 17) and quantum Hall effect measurements (Supplementary Fig. 22). Multiple control experiments (Supplementary Figs. 5-9) confirm that: (1) the $1^{\text {st }}$ layer growth is controlled by the traditional surface-mediated mechanism; 
(2) the $2^{\text {nd }}$ layer is fed by $\mathrm{C}$ that diffuses through the foil from the interior. Most notably, full coverage of the $1^{\text {st }}$ layer graphene on the exterior does not restrict growth of the $2^{\text {nd }}$ layer, whereas full coverage of the interior surface prevents through-diffusion and BLG growth.

BLG growth was only realized using $\mathrm{Cu}$ foils containing $\mathrm{O}$ impurities: when $\mathrm{OF}-\mathrm{Cu}$ was used under the same conditions no BLG was formed on the exterior surface, and the interior was densely covered with small hexagonal islands that merged to form a continuous film after 2 hours (Figs. 1e and 1f). In tests spanning a wide range of growth parameter settings, no BLG growth was ever observed on $\mathrm{OF}-\mathrm{Cu}$. One explanation for this difference is the role of oxygen in reducing graphene nucleation density ${ }^{20}$, which helps maintain a large area of exposed $\mathrm{Cu}$ on the interior. To test whether this is the only role of oxygen in promoting BLG growth, we increased $\mathrm{P}_{\mathrm{CH} 4}$ on $\mathrm{OR}-\mathrm{Cu}$ to achieve graphene nucleation density and growth rate similar to that observed above on OF-Cu (Supplementary Fig. 7). However, even with 70\% interior coverage of SLG within tens of minutes, the OR-Cu showed a large population of BLG on the exterior surface, whereas no detectable BLG was found on OF-Cu with similar interior surface SLG coverage. Therefore, O clearly plays a more critical role of activating the growth of BLG than simply increasing the area of exposed $\mathrm{Cu}$ on the interior. The above results also observed that reducing the $\mathrm{C}$ flux can reduce the BLG nucleation density, similar to the case of SLG growth on $\mathrm{Cu}$ 20,22,23. Indeed, by decreasing $\mathrm{P}_{\mathrm{CH} 4}$ (Figs. 2a-2c), we were able to decrease the nucleation density by more than 2 orders of magnitude and thus increase the BLG domain size from a few $\mu \mathrm{m}$ to $\sim 500 \mu \mathrm{m}$.

We visualize the time evolution of BLG growth by feeding ${ }^{12} \mathrm{CH}_{4}$ and ${ }^{13} \mathrm{CH}_{4}$ for alternating periods of $15 \mathrm{~min}$ and mapping the isotopic composition of the grown BLG by Raman spectroscopy. Figs. $2 \mathrm{f}$ and $2 \mathrm{j}$ show Raman maps of two representative BLG regions 
(optical images in Fig. 2e and 2i). The $1^{\text {st }}$ layer film shows isotopic rings characteristic of surface-limited growth, a result of diffusion of $\mathrm{C}$ radicals on the $\mathrm{Cu}$ surface to the edge of a growing domain ${ }^{24,25}$. Remarkably, we also observed isotopic rings in the $2^{\text {nd }}$ layer domains even though they are fed by $\mathrm{C}$ diffusion through the $\mathrm{Cu}$ foil. This is explained by (1) the extremely low C solubility [only a few parts per million (ppm) ${ }^{26}$ and (2) efficient diffusion both inside the $\mathrm{Cu}$ bulk and along the interface between $\mathrm{Cu}$ and the $1^{\text {st }}$ layer graphene (see below). This appears to be different from our prior observations, where the isotope rings in graphene were proposed as the result of surface-mediated mechanism oonly. ${ }^{24}$ The observation of isotopic rings synchronized with the sequential isotope-labeled dosing indicates that the $2^{\text {nd }}$ layer growth proceeds only at the high temperature, i.e., isothermally, rather than by $\mathrm{C}$ precipitation during cooling of $\mathrm{Cu}^{27}$ and distinct from the traditional precipitation-dominant growth of multilayer graphene on $\mathrm{Ni}$, in which isotopically labeled $\mathrm{C}$ mixes in the bulk prior to precipitation upon cooling and thus no isotopic rings are observed. ${ }^{8,24}$ Further discussions about the growth mechanism can be found in Supplementary Information.

Raman mapping can also disclose the origin of Bernal stacking and stacking disorder in CVD BLG. By using isotopic rings of the $2^{\text {nd }}$ layer domains, their nucleation sites were visualized (Figs. $2 \mathrm{~g}$ and $2 \mathrm{k}$ ). We then found that each $2^{\text {nd }}$ layer domain is always Bernal-stacked with the $1^{\text {st }}$ layer domain under which it initially nucleated. The $2^{\text {nd }}$ layer continues to grow as a single crystal, and therefore is Bernal-stacked as long as it remains under the same $1^{\text {st }}$ layer domain. Upon crossing a $1^{\text {st }}$ layer domain boundary (Figs. $2 \mathrm{j}$ and $2 \mathrm{k}$ ), it retains the same crystal orientation, and thus Bernal stacking is lost, as indicated by a decrease of the 2D FWHM to 25$40 \mathrm{~cm}^{-1}$ (Fig. 21). These characterizations clearly demonstrate that: (1) the lower energy of Bernal stacking is sufficient to ensure orientation during nucleation of the $2^{\text {nd }}$ layer; and (2) stacking 
disorder is a direct result of lateral growth of $2^{\text {nd }}$ layer domains across $1^{\text {st }}$ layer domain boundaries. Therefore, we expect that optimizing growth toward increasing the domain size in both layers should also increase Bernal stacking. Indeed, after analyzing the Raman images of a number of BLG regions under different growth conditions, we observed a significant increase in the area percentage of Bernal stacking in all BLG regions with decreasing $\mathrm{P}_{\mathrm{CH} 4}$, as shown in Fig. $2 \mathrm{~d}$. Thus, these results present a viable pathway toward scalable synthesis of large-area Bernalstacked BLG.

We propose that growth of the $2^{\text {nd }}$ layer graphene consists of the following major steps (Figs. 3a and 3b): (1) dehydrogenation of $\mathrm{CH}_{4}$ on the interior $\mathrm{Cu}$ surface, (2) $\mathrm{C}$ diffusion in $\mathrm{Cu}$ bulk and segregation to the exterior surface, (3) $\mathrm{C}$ diffusion along the $\mathrm{Cu}-1^{\text {st }}$ graphene layer interface, (4) nucleation of the $2^{\text {nd }}$ layer domains (the impurities or defects on the exterior surface are considered to be the preferential nucleation sites), and (5) $\mathrm{C}$ attachment to the domain edges for continuous growth. We stress that in (1) the $\mathrm{CH}_{4}$ must be completely dehydrogenated before entering the $\mathrm{Cu}$ bulk in the form of single $\mathrm{C}$ atoms. This is because large $\mathrm{C}$ species [e.g. C-C dimer, or $\left.\mathrm{CH}_{\mathrm{x}}(\mathrm{x}=1-4)\right]$ cannot be accommodated in the $\mathrm{Cu}$ lattice ${ }^{29}$, and their significant diffusion along $\mathrm{Cu}$ grain boundaries is unlikely as we did not see preferential formation of $2^{\text {nd }}$ layer domains near $\mathrm{Cu}$ grain boundaries (Supplementary Fig. 9).

However, the above scenario does not address the critical role of $\mathrm{O}$ in growth of $2^{\text {nd }}$ layer graphene. Further insight into the O-activated growth kinetics can be obtained from density functional theory (DFT) calculations. We first consider the dehydrogenation process of $\mathrm{CH}_{4}$ on a model $\mathrm{Cu}$ (111) surface (Fig. 3c). Each step of the $\mathrm{CH}_{4}$ dehydrogenation is endothermic, even on a catalytic $\mathrm{Cu}$ surface, creating a formidable kinetic energy barrier of over $4 \mathrm{eV}$, almost 3 times larger than the energy barrier governing SLG growth on $\mathrm{OF}-\mathrm{Cu}^{20,30} \cdot \mathrm{Cu}$ (111) surface defects, 
such as step edges, can only slightly lower the barrier by $\sim 0.6 \mathrm{eV}$. Since the growth temperature corresponds to only $\sim 0.1 \mathrm{eV}$, complete dehydrogenation of $\mathrm{CH}_{4}$ is essentially forbidden (relative to the growth of SLG) and the subsequent steps for $2^{\text {nd }}$ layer growth will therefore not happen. This explains why BLG domains are always absent on OF-Cu. However, when there are $\mathrm{O}$ atoms on the $\mathrm{Cu}$ surface, $\mathrm{C}-\mathrm{H}$ bond cleavage generally becomes easier since $\mathrm{O}$ can form a stable $\mathrm{OH}$ group with the $\mathrm{H}^{30}$ Indeed, in the presence of surface $\mathrm{O}$, the overall dehydrogenation barrier of $\mathrm{CH}_{4}$ dramatically decreases to $\sim 1.4 \mathrm{eV}$ (Fig. 3c), comparable to the barrier for SLG growth. Here we emphasize that the role of $\mathrm{O}$ in the BLG growth is distinct from its role in the growth of $\mathrm{SLG}^{20}$, in which $\mathrm{O}$ decreases the barrier for $\mathrm{CH}_{\mathrm{x}}$ attachment to graphene domain edges, but edge attachment process can still occur at a lower rate without O. In the BLG case, OF-Cu acts as an impenetrable barrier for $\mathrm{CH}_{\mathrm{x}}$. Oxygen is required for dehydrogenation of $\mathrm{CH}_{\mathrm{x}}$ so that atomic $\mathrm{C}$ can diffuse through the $\mathrm{Cu}$ foil for the $2^{\text {nd }}$ layer growth on the exterior surface. In other words, $\mathrm{O}$ opens up the kinetic pathway for BLG growth, a critical aspect that has been up to now completely overlooked ${ }^{7-19}$.

To evaluate whether the remaining proposed steps in the kinetic pathway of BLG growth can occur under normal growth conditions, we calculated the energy barriers for $\mathrm{C}$ atom hopping both in the $\mathrm{Cu}$ bulk (supplementary Fig. 19) and at the $\mathrm{Cu}-1^{\text {st }}$ graphene layer interface. For the former we obtained a barrier of only $\sim 1 \mathrm{eV}$, indicating that $\mathrm{C}$ can efficiently diffuse inside $\mathrm{Cu}$. This relatively low kinetic barrier is not contradictory to the low C solubility, which is a thermal equilibrium property. In the vicinity of the interface, a $\mathrm{C}$ atom is found to preferentially stay at the $\mathrm{Cu}$ subsurface (Fig. 3b, inset), with an energy $\sim 0.6 \mathrm{eV}$ lower than the interface, and $\sim 0.15 \mathrm{eV}$ lower than that in $\mathrm{Cu}$ bulk interstitial sites (Supplementary Table 1). Therefore $\mathrm{C}$ atoms accumulate near the $\mathrm{Cu}$ surface rather than in the bulk. More interestingly, the $1^{\text {st }}$ graphene layer 
reduces the barrier for $\mathrm{C}$ atom hopping along the subsurface sites, from $0.92 \mathrm{eV}$ on a bare $\mathrm{Cu}$ (111) substrate to $0.45 \mathrm{eV}$ (Fig. 3d and supplementary Table 1). This is due to 'lifting' of the surface $\mathrm{Cu}$ atoms by the weak chemical bonds with the $1^{\text {st }}$ graphene layer (Fig. 3d). The subsurface diffusion therefore has a much lower barrier than other steps in the $2^{\text {nd }}$ layer graphene growth, which reduces the possibility of new island nucleation and facilitates growth of large and uniform $2^{\text {nd }}$ layer domains, as demonstrated by the isotopic rings of the $2^{\text {nd }}$ layer (Fig. 2).

To assess the electrical quality of the Bernal-stacked BLG, the BLG was encapsulated between a few-nm thick hexagonal boron nitride (h-BN) flakes, which act as back- and top-gate dielectrics (Fig. 4a and Supplementary Fig. 21). The resistivity as a function of back-gate voltage, $\mathrm{V}_{\mathrm{bg}}$, is shown in Fig. $4 \mathrm{~b}$. The curve exhibits a narrow and symmetric resistivity peak (FWHM of Dirac peak $\sim 1.5 \mathrm{~V}$ ), with the charge neutrality point $\mathrm{V}_{\mathrm{bg}}=-0.1 \mathrm{~V}$. These features suggest low densities of charged impurities and defects, and hence high crystalline quality. The conductivity versus $\mathrm{V}_{\mathrm{bg}}$ (Fig. 4b) exhibits a fairly symmetric and sub-linear behavior. The mobility is found to be $60,000 \mathrm{~cm}^{2} \mathrm{~V}^{-1} \mathrm{~s}^{-1}$ at $1.7 \mathrm{~K}$ and $20,000 \mathrm{~cm}^{2} \mathrm{~V}^{-1} \mathrm{~s}^{-1}$ at $300 \mathrm{~K}$. A bandgap was induced in the BLG by application of an out-of-plane electric field (by voltage difference between the top- and back-gates). At 1.7K, an on/off ratio of more than 40,000 is observed (Fig. $4 c$ ), more than 3 orders of magnitude higher than previously reported for CVD BLG ${ }^{11-14}$ and comparable to that achieved for exfoliated $\mathrm{BLG}^{3}$. The bandgap values were extracted at each fixed electric field by fitting the log of resistivity versus inverse temperature (Fig. 4e) according to the Arrhenius relation with a simple thermal activation mechanism, i.e., $\mathrm{R} \sim \exp \left(\Delta \mathrm{E} / \mathrm{k}_{\mathrm{B}} \mathrm{T}\right)$, where $\mathrm{R}$ is the resistivity and $\Delta \mathrm{E}$ is the bandgap $^{32}$. The extracted bandgap values are in agreement with both theoretical predictions and infrared spectroscopy measurements ${ }^{2}$ (Fig. 4f). 
The maximum bandgap is $103 \mathrm{meV}$ at a transverse field of $0.9 \mathrm{~V} / \mathrm{nm}$, limited only by the field supported by the gate dielectrics.

In conclusion, our multiple experimental and theoretical results disclose that in a LPCVD growth environment, $\mathrm{O}$ impurities on the $\mathrm{Cu}$ surface play a key role in activating hydrocarbon molecular dissociation that allows the ultimate conversion of $\mathrm{CH}_{x}$ to $\mathrm{C}$ atoms that dissolve into the $\mathrm{Cu}$ foil, without which BLG growth on $\mathrm{Cu}$ could not proceed. Complete understanding of the growth mechanism enables the growth of large and single-crystal Bernal-stacked BLG domains. Furthermore, the BLG shows superior electrical quality comparable to exfoliated BLG, thus promising future BLG-based electronics and photonics.

1. Geim, A. K. \& Novoselov, K. S. The rise of graphene. Nat. Mater. 6, 183-191 (2007).

2. Zhang, Y. et al. Direct observation of a widely tunable bandgap in bilayer graphene. Nature 459, 820-823 (2009).

3. Yan, J. et al. Dual-gated bilayer graphene hot-electron bolometer, Nat. Nanotechnol. 7, 472478 (2012).

4. Yu, Q. et al. Control and characterization of individual grains and grain boundaries in graphene grown by chemical vapor deposition. Nat. Mater. 10, 443-449 (2011).

5. Yazyev, O. V. \& Louie, S. G. Electronic transport in polycrystalline graphene. Nat. Mater. 9, 806-809 (2010).

6. Kim, K. et al. Raman spectroscopy study of rotated double-layer graphene: misorientation angle dependence of electronic structure. Phys. Rev. Lett. 108, 246103 (2012).

7. Batzill, M. The surface science of graphene: metal interfaces, CVD synthesis, nanoribbons, chemical modifications, and defects. Surf. Sci. Rep. 67, 83-115 (2012).

8. Kim, K.S. et al. Large-scale pattern growth of graphene films for stretchable transparent electrodes. Nature 457, 706-710 (2010).

9. Weatherup, R. S. et al. Interdependency of subsurface carbon distribution and graphenecatalyst interactions. J. Am. Chem. Soc. 136, 13698-13708 (2014). 
10. Mattevi, C., Kim, H. \& Chhowalla, M. A review of chemical vapour deposition of graphene on copper. J. Mater. Chem. 21, 3324-3334 (2011).

11. Lee, S., Lee, K. \& Zhong, Z. Wafer scale homogeneous bilayer graphene films by chemical vapor deposition. Nano Lett. 10, 4702-4707 (2010).

12. Liu, W. et al. Controllable and rapid synthesis of high-quality and large-area Bernal stacked bilayer graphene using chemical vapor deposition. Chem. Mater. 26, 907-915 (2014).

13. Zhou, H. et al. Chemical vapor deposition growth of large single crystals of monolayer and bilayer graphene, Nat. Commun. 4, 2096 (2013) doi:10.1038/ncomms3096.

14. Yan, K., Peng, H., Zhou, Y., Li, H. \& Liu, Z. Formation of bilayer Bernal graphene: layerby-layer epitaxy via chemical vapor deposition. Nano Lett. 11, 1106-1110 (2011).

15. Fang, W. et al. Asymmetric growth of bilayer graphene on copper enclosures using low pressure chemical vapor deposition. ACS Nano 8, 6491-6499 (2014).

16. Sun, Z. et al. Large-area Bernal-stacked bi-, tri-, and tetralayer graphene. ACS Nano 6 , 9790-9796 (2012).

17. Liu, L. et al. High-yield chemical vapor deposition growth of high-quality large-area ABstacked bilayer graphene. ACS Nano 6, 8241-8249 (2012).

18. Zhao, P. et al. Equilibrium chemical vapor deposition growth of Bernal-stacked bilayer graphene. ACS Nano, 8, 11631-11638 (2014).

19. Gan, L. et al. Grain size control in the fabrication of single-crystal bilayer graphene structures. Nanoscale, 7, 2391-2399 (2015).

20. Hao, Y. et al. The role of surface oxygen in the growth of large single-crystal graphene on copper, Science 342, 720-723 (2013).

21. Hao, Y. et al. Probing layer number and stacking order of few-layer graphene by Raman spectroscopy. Small 6, 195-200 (2010).

22. Vlassiouk, I. et al. Graphene nucleation density on copper: fundamental role of background pressure. J. Phys. Chem. C, 117, 18919-18926 (2013)

23. Yan, Z. et al. Toward the synthesis of wafer-scale single-crystal graphene on copper foils. ACS Nano, 6, 9110-9117 (2012).

24. Li, X., Cai, W., Colombo, L. \& Ruoff, R. S. Evolution of graphene growth on Ni and Cu by carbon isotope labeling. Nano Lett. 9, 4268-4272 (2009). 
25. Kim, H. et al. Activation energy paths for graphene nucleation and growth on Cu. ACS Nano, 6, 3614-3623 (2012).

26. López, G. A. \& Mittemeijer, E. J. The solubility of C in solid Cu. Scr. Mater. 51, 1-5 (2004).

27. Kidambi, P. R. et al. Observing graphene grow: catalyst-graphene interactions during scalable graphene growth on polycrystalline copper. Nano Lett. 13, 4769-4778 (2013).

28. Fang, W. et al. Rapid identification of stacking orientation in isotopically labeled chemicalvapor grown bilayer graphene by Raman spectroscopy, Nano Lett. 13, 1541-1548 (2013).

29. Riikonen, S., Krasheninnikov, A. V., Halonen, L. \& Nieminen, R. M. The role of stable and mobile carbon adspecies in copper-promoted graphene growth, J. Phys. Chem. C 116, 58025809 (2012).

30. Zhang, W., Wu, P., Li, Z. \& Yang, J. First-principles thermodynamics of graphene growth on Cu surfaces J. Phys. Chem. C, 115, 17782-17787 (2011).

31. Alstrup, I., Chorkendorff, I. \& Ullmann, S. The interaction of $\mathrm{CH}_{4}$ at high temperatures with clean and oxygen precovered $\mathrm{Cu}(100)$. Surf. Sci. 264, 95-102 (1992).

32. Taychatanapat, T. \& Jarillo-Herrero, P. Electronic transport in dual-gated bilayer graphene at large displacement fields Phys. Rev. Lett.105, 166601 (2010).

Supplementary Information is linked to the online version of the paper.

Acknowledgements We thank John B. Goodenough (University of Texas), Feng Wang and Long Ju (University of California), Eric Stach and Dong Su (Brookhaven National Laboratory), Gabriel A. López (University of Basque Country), Eric J. Mittemeijer (MPI, Germany), Zhenyu Zhang (University of Science and Technology of China), N. C. Bartelt (Sandia Laboratories), Yili Gao, and Jun Yin (Nanjing University of Aeronautics and Astronautics) for valuable discussions and/or technical assistance. We thank K. Watanabe and T. Taniguchi for providing h-BN substrates. Work at Columbia University was supported by Office of Naval Research (ONR) -N000141310662 and the Nanoelectronics Research Initiative (NRI) through the Institute 
for Nanoelectronics Discovery and Exploration (INDEX). Work at Austin was supported by the South West Academy of Nanolectronics (SWAN) of the NRI. Work at Sandia was supported by the Office of Basic Energy Sciences, Division of Materials and Engineering Sciences, U. S. Department of Energy under Contract No. DE-AC04-94AL85000. Work at Rice University was supported by the ONR and NSF's Chemical, Bioengineering, Environmental, and Transport Systems Division. Work at NREL was supported by U.S. DOE under Contract No. DE-AC3608GO28308, and used the NREL Peregrine Supercomputer. Work at Hong Kong was supported by Hong Kong University of Science and Technology under award RPC11EG39. H. C. acknowledges support from NSF DMR-1122603 and from ONR-N00014-14-1-0330. C. T. acknowledges support from a National Defense Science and Engineering Graduate (NDSEG) Fellowship - FA9550-11-C-0028. R.S.R. was supported by IBS-R019-D1.

Author Contributions Y.F.H., R.S.R., and L.C. conceived of the experiments, and Y.F.H. designed and performed all the growth experiments and graphene transfer for Raman and electrical measurements. R.S.R., J.H. and L.C. supervised the project. Y.F.H., H.C., L.C., J.H., K.F.M., X.W., C.T., J.W.S., and R.S.R. analyzed experimental results and data. L.W., J.H., P.K., and C.R.D. performed electrical measurements. Y.L. and B.I.Y. performed DFT calculations. K.F.M. and S.N. performed LEEM and LEED experiments. T. J. performed the focus ion beam cutting. T.L., J.X., and W.Y. performed the gas exchange rate calculations. Y.F.H., H.C., J.H., R.S.R., and L.C. wrote the manuscript. All co-authors revised and commented on the manuscript. 
Figures
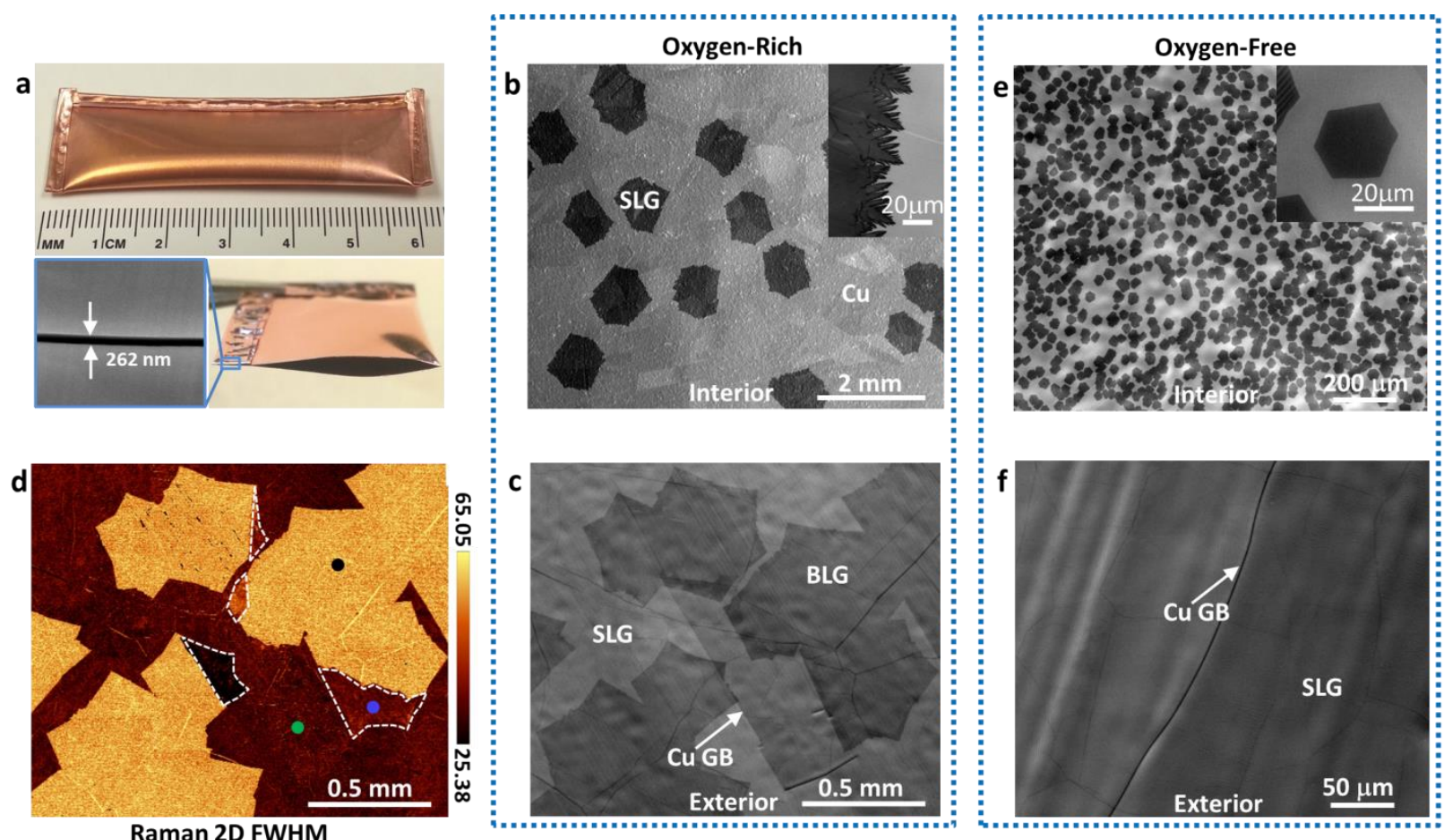

Figure 1 | Growth of Large BLG domains on the exterior surface of an OR-Cu pocket. a, Optical image of the $\mathrm{Cu}$ pocket. The lower inset shows the optical and corresponding SEM images of the cut-edge cross section. $\mathbf{b}$ and $\mathbf{c}$, SEM images of graphene domains grown on the interior and exterior surfaces of an OR-Cu pocket, respectively. The growth was proceeded at $1035^{\circ} \mathrm{C}$ and $\mathrm{P}_{\mathrm{CH} 4}=1 \times 10^{-3}$ Torr. Inset in $\mathbf{b}$ shows the dendritic edge of the domains. $\mathbf{d}$, Raman image of 2D band FWHM of the same area as in c. The bright areas indicate Bernal stacking. Raman spectra at different points are shown in Fig. S15. e and f, Graphene domains/films grown on the interior and exterior surfaces of an $\mathrm{OF}-\mathrm{Cu}$ pocket, respectively. Inset in e shows the compact hexagonal domains. $\mathrm{Cu}$ grain boundary (GB) lines are marked by arrows in $\mathbf{c}$ and $\mathbf{f}$. 

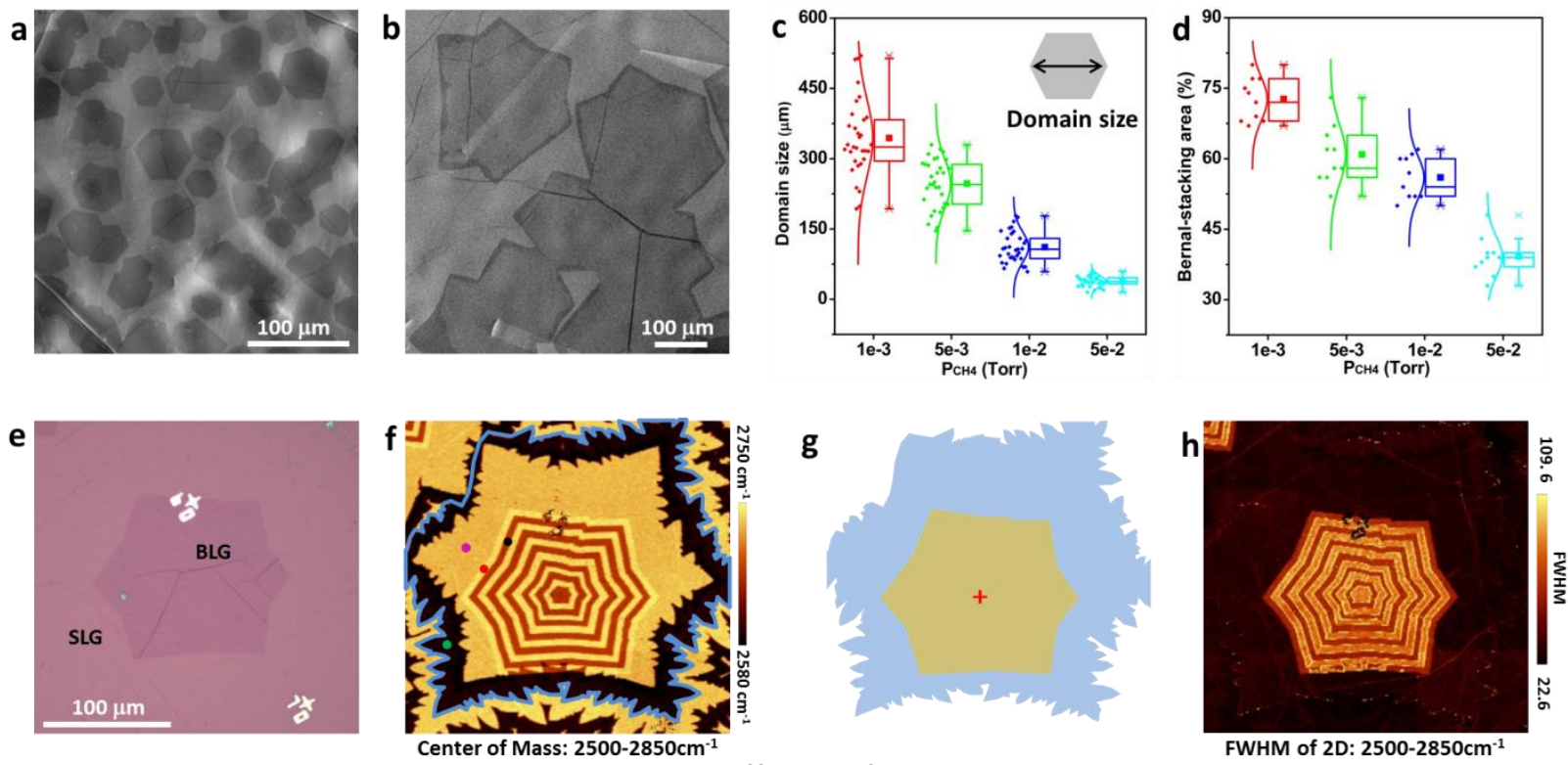

${ }^{12} \mathrm{C} \overbrace{}^{13} \mathrm{C}^{12} \mathrm{C}{ }^{13} \mathrm{C}^{12} \mathrm{C}{ }^{13} \mathrm{C}^{12} \mathrm{C}{ }^{13} \mathrm{C}{ }^{12} \mathrm{C}^{13} \mathrm{C}^{12} \mathrm{C}^{13} \mathrm{C}{ }^{12} \mathrm{C}$
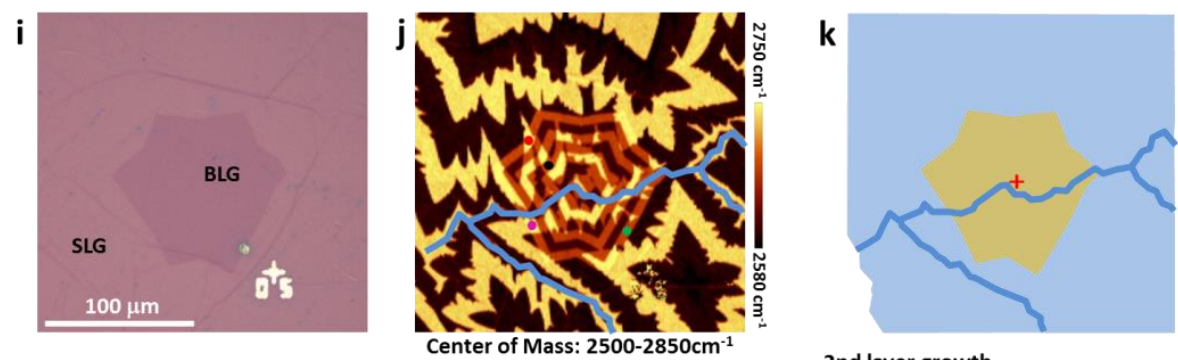

2nd layer growth

${ }^{12} \mathrm{C}{ }^{13} \mathrm{C}{ }^{12} \mathrm{C}{ }^{13} \mathrm{C}^{12} \mathrm{C}{ }^{13} \mathrm{C}{ }^{12} \mathrm{C}^{13} \mathrm{C}{ }^{12} \mathrm{C}{ }^{13} \mathrm{C}^{12} \mathrm{C}{ }^{13} \mathrm{C}{ }^{12} \mathrm{C}$

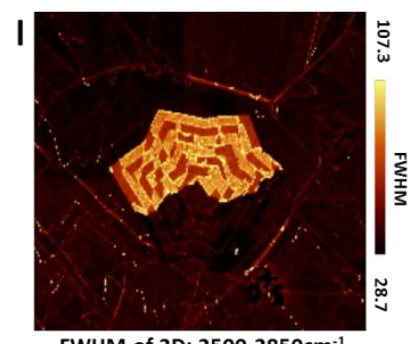

FWHM of 2D: $2500-2850 \mathrm{~cm}^{-1}$

Figure 2 | BLG domain size and stacking order control. a and b, SEM images of BLG domains grown at $\mathrm{P}_{\mathrm{CH} 4}=5 \times 10^{-2}$ Torr and $5 \times 10^{-3}$ Torr, respectively. $\mathbf{c}$ and $\mathbf{d}$, Boxplots of $2^{\text {nd }}$ layer domain size and Bernal-stacked area percentage as a function of $\mathrm{P}_{\mathrm{CH}_{4}}$, respectively. e and $\mathbf{i}$, Optical images and $\mathbf{f}, \mathbf{h}, \mathbf{j}$, and $\mathbf{l}$, the corresponding Raman images. Note that 2D-band FWHM of $50-55 \mathrm{~cm}^{-1}$ (or $100-110 \mathrm{~cm}^{-1}$ for isotope-labeled regions ${ }^{28}$ ) is the characteristic of Bernal stacking. Each isotopic cycle is $15 \mathrm{~min}$ at $1035^{\circ} \mathrm{C}$ and $\mathrm{P}_{\mathrm{CH} 4} \sim 2 \times 10^{-3}$ Torr. Raman spectra at different points are shown in Fig. S15. $\mathbf{g}$ and $\mathbf{k}$, Schematics of $2^{\text {nd }}$ layer nucleation sites (red crosses). The thick blue lines in $\mathbf{k}$ are $1^{\text {st }}$ graphene layer domain boundaries. 

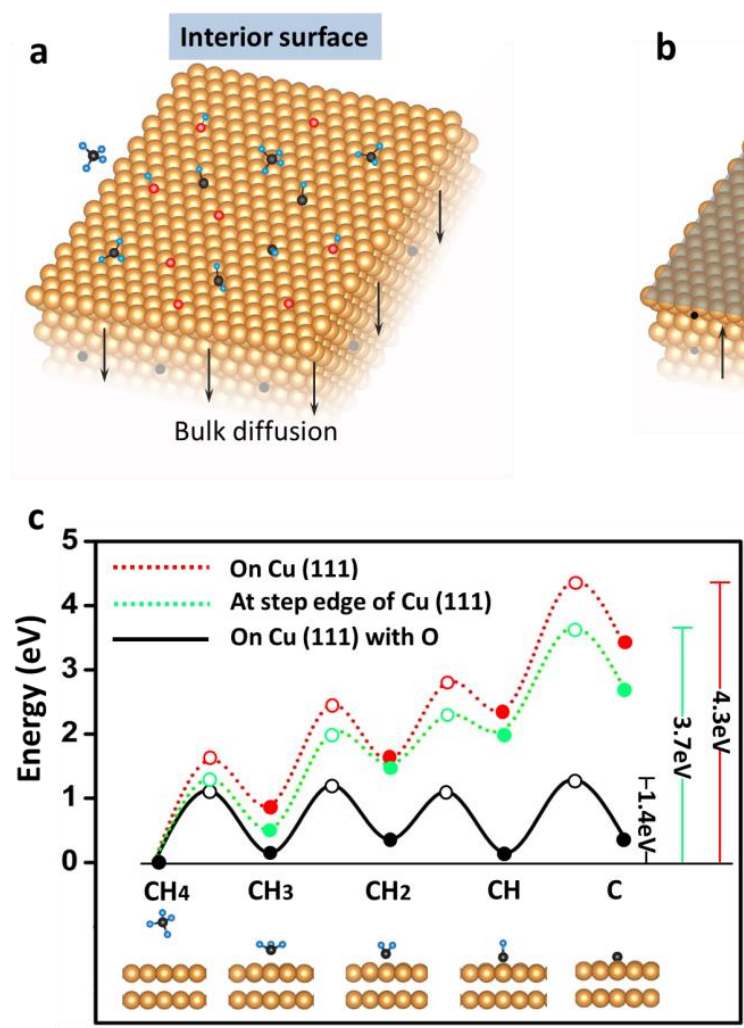

Reaction pathway
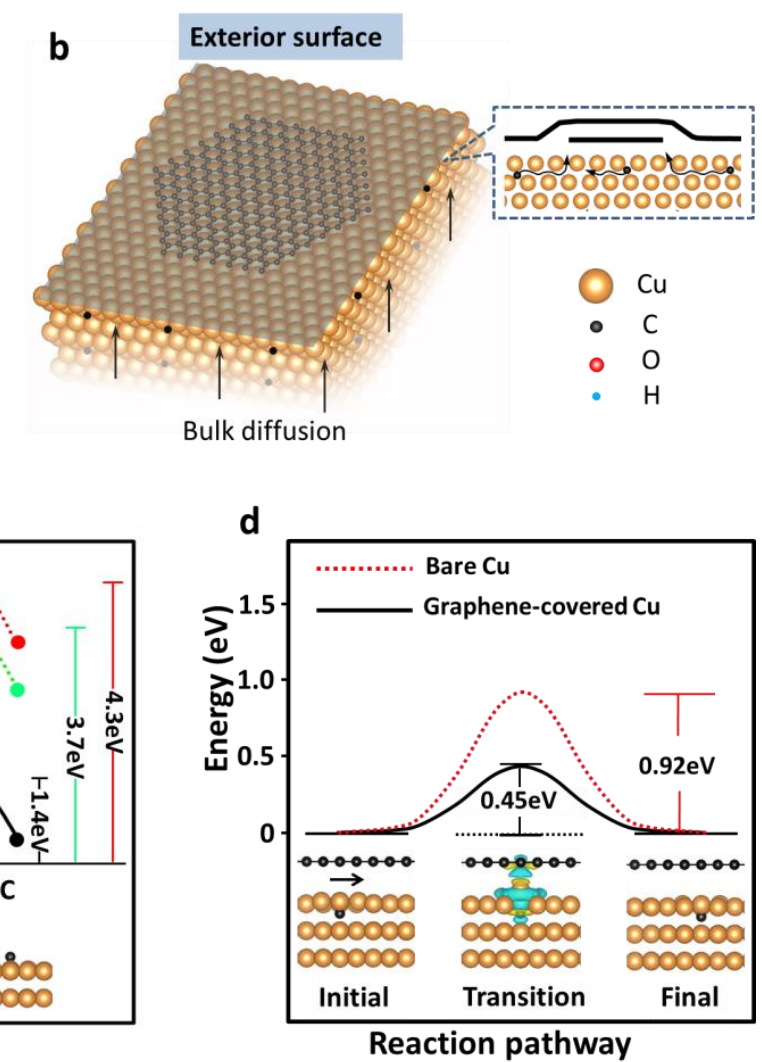

Reaction pathway

Figure 3 | O-activated growth mechanism and DFT calculations. a and b, Schematics of the dynamic processes from interior to exterior surfaces of the $\mathrm{Cu}$ pocket. c, Dehydrogenation processes and the associated barriers on ideal $\mathrm{Cu}(111)$ surface, step edges of $\mathrm{Cu}(111)$, and $\mathrm{Cu}(111)$ with surface $\mathrm{O}$. Insets show the equilibrium positions of $\mathrm{CH}_{\mathrm{x}}$ radicals on ideal $\mathrm{Cu}(111)$. d, The subsurface diffusion and the associated barriers on graphene-covered and bare $\mathrm{Cu}$, respectively. The charge density difference of the "lifted-up" $\mathrm{Cu}$ atom at the transition state, revealed by the colored "iso-surface" (details in Supplementary Information), suggests a weak bond formed between the $\mathrm{Cu}$ atom and the graphene layer. 
a
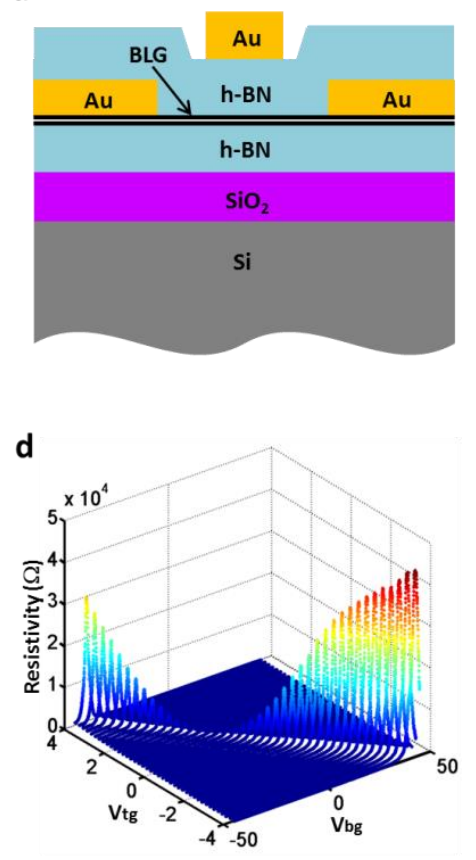

b
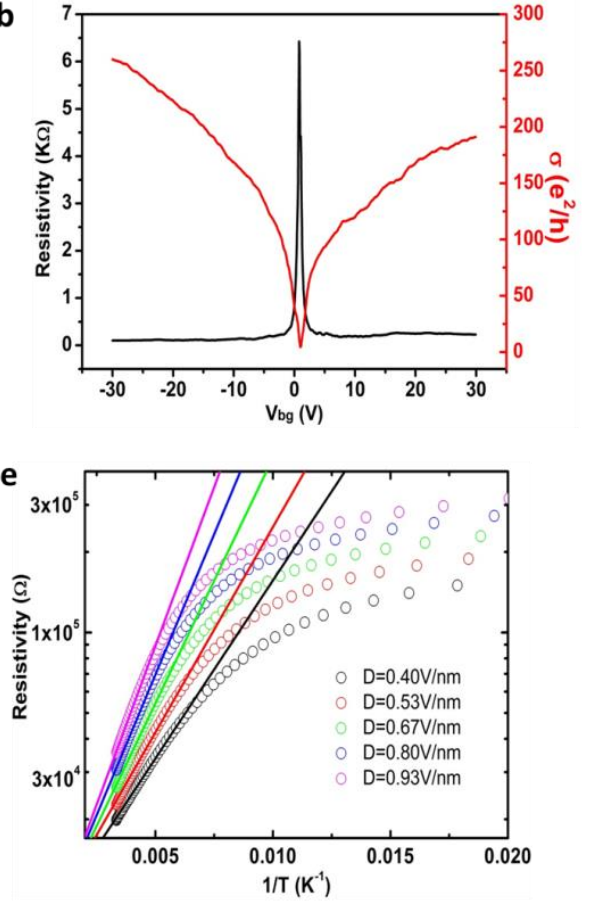
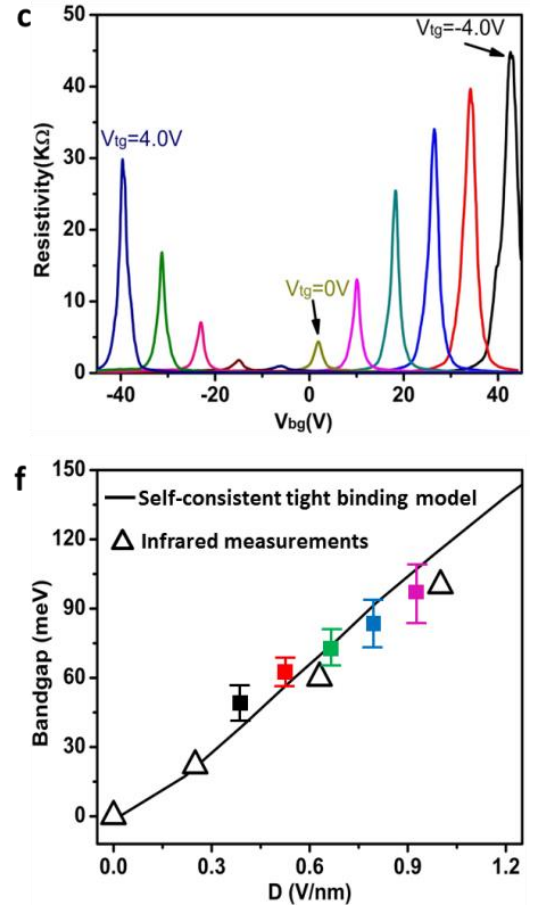

Figure 4 | Electrical transport in Bernal-stacked BLG. a, Schematic of the BLG device. b, Plots of resistivity and corresponding conductivity as a function of $\mathrm{V}_{\mathrm{bg}}$ at $1.7 \mathrm{~K}$. c, Resistivity as a function of $\mathrm{V}_{\mathrm{bg}}$ at different $\mathrm{V}_{\mathrm{tg}}$. The traces are taken with $0.8 \mathrm{~V}$ steps in $\mathrm{V}_{\mathrm{tg}}$ from $-4 \mathrm{~V}$ to $4 \mathrm{~V}$. d, Three-dimensional color plot of the resistivity vs. $\mathrm{V}_{\mathrm{tg}}$ and $\mathrm{V}_{\mathrm{bg}}$ at $1.7 \mathrm{~K}$. e, Resistivity as a function of inverse temperature from 50-300 K. f, Bandgap values extracted from e and compared with theoretical prediction and infrared measurements ${ }^{2}$. 